\title{
Safe and effective prescription of exercise in acute exacerbations of chronic obstructive pulmonary disease: Rationale and methods for an integrated knowledge translation study
}

\author{
Pat G Camp PT PhD 1,2,3,4, W Darlene Reid PT PhD ${ }^{3,4,5}$, Cristiane Yamabayashi PT PhD ${ }^{1,4,5}$, Dina Brooks PT PhD ${ }^{6}$,
} Donna Goodridge RN PhD ${ }^{7}$, Frank Chung PT MSc ${ }^{8}$, Darcy D Marciniuk MD FRCPC ${ }^{9}$,

Andrea Neufeld $\mathrm{BSc}^{3}$, Alison Hoens PT $\mathrm{MSc}^{2,3}$

\begin{abstract}
PG Camp, WD Reid, C Yamabayashi, et al. Safe and effective prescription of exercise in acute exacerbations of chronic obstructive pulmonary disease: Rationale and methods for an integrated knowledge translation study. Can Respir J 2013;20(4):281-284.
\end{abstract}

BACKGROUND: Patients hospitalized with an acute exacerbation of chronic obstructive pulmonary disease (AECOPD) engage in low levels of activity, putting them at risk for relapse and future readmissions. There is little direction for health care providers regarding the parameters for safe exercise during an AECOPD that is effective for increasing activity tolerance before discharge from hospital, especially for patients with associated comorbid conditions.

OBJECTIVE: To report the rationale for and methods of a study to develop evidence-informed care recommendations that guide health care providers in the assessment, prescription, monitoring and progression of exercise for patients hospitalized with AECOPD.

METHODS: The present study was a multicomponent knowledge translation project incorporating evidence from systematic reviews of exercise involving populations with chronic obstructive pulmonary disease and/or common comorbidities. A Delphi process was then used to obtain expert opinion from clinicians, academics and patients to identify the parameters of safe and effective exercise for patients with AECOPD.

RESULTS: Clinical decision-making tool(s) for patients and practitioners supported by a detailed knowledge dissemination, implementation and evaluation framework.

CONCLUSION: The present study addressed an important knowledge gap: the lack of availability of parameters to guide safe and effective exercise prescription for hospitalized patients with AECOPD, with or without comorbid conditions. In the absence of such parameters, health care professionals may adopt an 'activity as tolerated' approach, which may not improve physical activity levels in their patients. The present study synthesizes the best available evidence and expert opinion, and will generate decision-making tools for use by patients and their health care providers.

Key Words: Chronic obstructive pulmonary disease; Exacerbations; Exercise; Knowledge translation

A ccording to a 2010 report by the Canadian Thoracic Society (1), acute respiratory exacerbations of chronic obstructive pulmonary disease (AECOPD) are the number one cause of ambulatory care hospitalizations in Canada and are estimated to cost the Canadian health care system $\$ 1.5$ billion per year. On average, patients with an AECOPD often experience repeated hospitalizations that are associated with lengths of stay of nine to 10 days (2). A common sequela of these exacerbations is profoundly reduced activity levels, resulting in

\section{La prescription sécuritaire et efficace d'exercice lors d'exacerbations aiguës de la maladie pulmonaire obstructive chronique : le fondement et la méthodologie d'une étude intégrée de transfert du savoir}

\begin{abstract}
HISTORIQUE : Les patients hospitalisés en raison d'une exacerbation aiguë de la maladie pulmonaire obstructive chronique (EAMPOC) font peu d'activité, ce qui les rend vulnérables à une rechute et à de futures réhospitalisations. Les dispensateurs de soins ont peu de directives sur les paramètres d'exercice sécuritaire pendant une EAMPOC, surtout lorsque les patients ont des maladies comorbides connexes.

OBJECTIF : Rendre compte du fondement et de la méthodologie d'une étude pour élaborer des recommandations de soins fondées sur des données probantes qui orientent les dispensateurs de soins dans l'évaluation, la prescription, la surveillance et la progression de l'exercice chez les patients hospitalisés en raison d'une EAMPOC.

MÉTHODOLOGIE : La présente étude était un projet de transfert du savoir à multiples volets intégrant des données probantes tirées d'analyses systématiques de l'exercice dans des populations ayant une maladie pulmonaire obstructive chronique ou des comorbidités courantes. Un processus Delphi a ensuite été utilisé pour obtenir les avis d'experts de cliniciens, d'universitaires et de patients, afin de déterminer les paramètres d'exercice sécuritaire et efficace pour les patients ayant une EAMPOC.

RÉSULTATS : Les outils de prise de décision clinique pour les patients et les praticiens étaient soutenus par une diffusion, une mise en œuvre et un cadre d'évaluation détaillés du savoir.

CONCLUSION : La présente étude traitait d'une lacune importante : l'absence de paramètres disponibles pour orienter une prescription d'exercice sécuritaire et efficace chez les patients hospitalisés ayant une EAMPOC, qu'ils présentent ou non des maladies comorbides. En l'absence de tels paramètres, les professionnels de la santé peuvent adopter une approche d'" activité selon la tolérance ", qui n'améliorera peut-être pas le taux d'activité de leurs patients. La présente étude synthétise les meilleurs données probantes et avis d'experts disponibles et permettra de créer des outils de prise de décision que pourront utiliser les patients et leurs dispensateurs de soins.
\end{abstract}

deconditioning and an increased risk for future hospitalizations and increased mortality (3). Compounding this problem is that patients with chronic obstructive pulmonary disease (COPD) often have other chronic comorbidities that impact their physical activity and their ability to recover from an AECOPD, including frailty, heart disease and/or osteoarthritis.

Physical activity is body movement produced by the skeletal muscles that requires energy expenditure. One approach to increase

${ }^{1}$ James Hogg Research Centre, St Paul's Hospital; ${ }^{2}$ Providence Health Care; ${ }^{3}$ Department of Physical Therapy; ${ }^{4}$ Institute of Heart and Lung

Health; ${ }^{5}$ Vancouver Coastal Health Research Institute, University of British Columbia, Vancouver, British Columbia; ${ }^{6}$ Department of Physical

Therapy, University of Toronto, Toronto, Ontario; ${ }^{7}$ College of Nursing, University of Saskatchewan, Saskatoon, Saskatchewan; ${ }^{8}$ Department

of Physical Therapy, Fraser Health Authority, Burnaby Hospital, Burnaby, British Columbia; ${ }^{9}$ Division of Respirology, Critical Care and Sleep

Medicine, University of Saskatchewan, Saskatoon, Saskatchewan

Correspondence: Dr Pat G Camp, James Hogg Research Centre, University of British Columbia, 1081 Burrard Street,

Vancouver, British Columbia V6Z 1Y6. Telephone 604-806-9144, e-mail pat.camp@hli.ubc.ca 


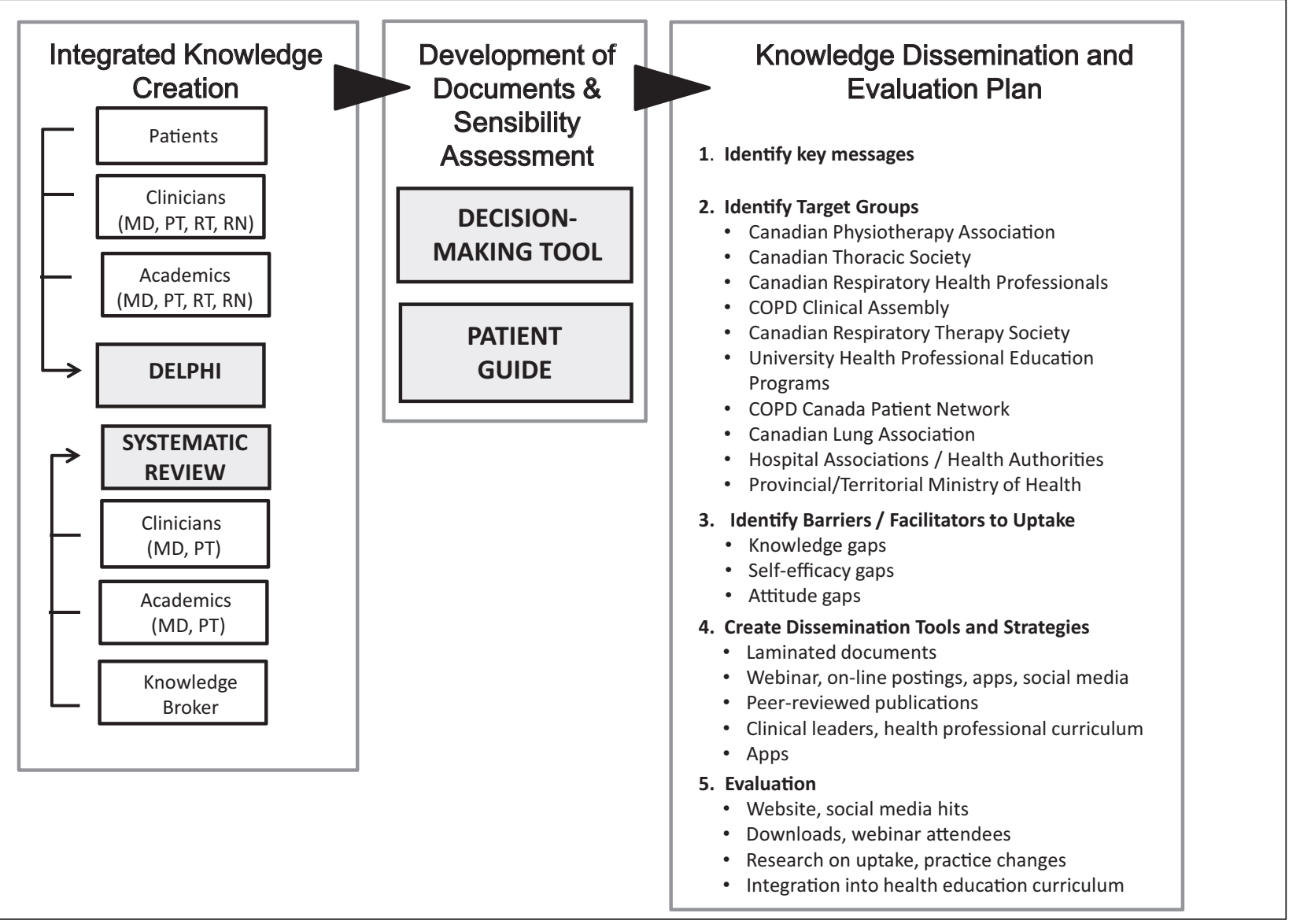

Figure 1) Knowledge translation development, dissemination and evaluation plan. COPD Chronic obstructive pulmonary disease; MD Physician; PT Physiotherapist; RN Registered nurse; RT Respiratory therapist

physical activity is to develop structured exercise programs that target the individual physical limitations of the patient. There is little information to guide health care providers in prescription parameters for safe exercise during an AECOPD that is effective for increasing activity tolerance before discharge from hospital. Although a systematic review has assessed the benefits of in-hospital or early rehabilitation for AECOPD patients (4), the studies in that review did not provide details regarding parameters to ensure safety and had limited information regarding the exercise provided or enrolled COPD patients without comorbidities. In the absence of practical clinical guidelines, health care professionals are liable to err on the side of caution and adopt a typically conservative 'activity as tolerated' approach for their patients and avoid prescribing a structured exercise program designed to address their patient's individual physical limitations. This may insufficiently address the poor activity tolerance, resulting in deconditioning and worse patient outcomes after discharge.

The present article outlines the methodology and rationale of a knowledge translation (KT) study designed to address this knowledge gap. The study was devised to develop an evidence-informed decisionmaking tool that guides health care providers in the assessment, prescription and monitoring of exercise for hospitalized patients with an AECOPD. Using an integrated KT approach, the present study aimed to incorporate the knowledge and perspectives of the highest quality literature, expert opinion and patient perspectives. The objectives of the present study were to:

1. Conduct a systematic review of the literature to identify the parameters of safe and effective exercise prescription for hospitalized patients with an AECOPD;

2. Conduct a modified Delphi process with multidisciplinary clinical experts, academics and patients, with representation from across Canada;
3. Develop a decision-making tool for clinicians based on the findings of the previous two objectives, with accompanying patient education, to guide the delivery of safe and effective exercise for patients with an AECOPD; and

4. Create and apply a knowledge dissemination, implementation and evaluation framework to facilitate the translation of this knowledge into clinical practice.

\section{METHODS}

The present KT study was a multistep project (Figure 1) that fully integrated the knowledge and experience of clinical experts, patients and academic health care professionals, with high-quality evidence from published research. The project team was interdisciplinary and represented health care professionals and researchers from the physiotherapy, nursing and medical professions, as well as a patient representative. The study was funded by a grant from the Canadian Institutes of Health Research (CIHR No: 226908).

Step one: the systematic review

The first step of the study was to conduct a systematic review (SR) of the literature. Multiple SRs have synthesized evidence on the benefits of exercise in chronic conditions. However, it can be difficult to extract the key messages from SRs that vary in their study question, and the quality and completeness of the review. In this circumstance, an SR of SRs (SR-SR) is necessary. SR-SR have been conducted in many health areas, including exercise (5), smoking cessation (6) and sleep disorders (7). The benefit of this approach is that it enables the opportunity to compare and contrast the quality, findings and the strength of the conclusions from the individual reviews. The SR-SR has been completed and recently published (8).

The SR was conducted based on the principles outlined by the Cochrane Collaboration. The objective of the SR-SR was to 
determine the effectiveness and safety of exercise for COPD and for typical comorbid conditions associated with COPD. Multiple databases including MEDLINE/PubMed, Embase and PEDro were searched to the end of July 2011. Reference lists of articles and the grey literature were also searched. Population keywords (eg, "chronic obstructive pulmonary disease", "osteoporosis" and "heart failure") were paired with intervention terms related to physical activity and exercise (eg, "exercise", "activity" and "mobility"). Study inclusion criteria were: adult participants with a diagnosis of AECOPD and/or other relevant acute care hospital patient population; an intervention of physical activity or exercise; physical fitness outcomes; and publication in English. The other relevant acute care hospital patient populations included were pneumonia, heart failure, cardiovascular disease, acute medical conditions, asthma, bronchiectasis, acute bronchitis, osteoarthritis, osteoporosis, diabetes and older adults. Titles and abstracts were screened independently by two reviewers (PGC, WDR) with differences resolved by consensus. Full-text articles of selected citations were screened for inclusion by two reviewers and, in the event of a disagreement, a third reviewer assisted with decision making for inclusion.

The articles were categorized according to the 12 disease conditions (AECOPD plus 11 comorbid conditions). One reviewer conducted the full data abstraction, which was verified by a second reviewer. Discrepancies were resolved through discussion or with a third reviewer. Tables were generated from the extracted data to describe characteristics of the studies within each review, including subject characteristics, the intervention and the outcomes. The methodological quality of articles were assessed by two reviewers using A Measurement Tool to Assess Systematic Reviews (AMSTAR) (9) rating scale for systematic reviews. A synthesis of the findings for each disease condition was developed in conjunction with the quality of the evidence. Complete details of the SR-SR and the summaries can be found in the full publication (8).

\section{Step two: the Delphi process}

Based on the examination of previous reviews, it was recognized that the systematic review conducted in Step one may not have elicited the level of detail of the necessary exercise parameters. In situations in which the empirical evidence is incomplete, eliciting and integrating the opinions of experts is important. The Delphi technique is one well-recognized method for eliciting and synthesizing these opinions. It consists of a series of sequential questionnaires combined with anonymized structured feedback. This anonymization minimizes the potential for dominating influence from any group members. The Delphi technique is not a scientific method to create new knowledge - it is a method for making the best use of available evidence such as high-quality research or the wisdom of experts.

Identification of the panelists: A three-round Delphi survey of 30 experts from the following target groups was conducted: clinical and academic experts from the health disciplines of physiotherapy (cardiorespiratory), medicine (respirology or internal medicine), nursing (acute care or intensive care) and respiratory therapy (acute care or intensive care); and patient experts. Panelists were selected from western Canada, eastern Canada and the Atlantic provinces, and from metropolitan, urban and rural settings. Three rounds are considered to be an adequate number to elicit the necessary information without leading to participant fatigue. Inclusion criteria for a clinical expert were: five or more years of clinical experience; currently working in an acute care hospital; and regularly treating patients with AECOPD. Inclusion criteria for an academic expert were five or more years of research experience; or one research publication in COPD; or currently teaching in exercise physiology or COPD. Patients had a self-reported diagnosis of COPD and at least one AECOPD within the past two years. All panelists agreed to complete the Delphi process and provided informed, signed consent. This study was approved by the Providence Health Research Ethics Board.
Delphi process - rounds 1, 2 and 3: The purpose of the first round was to generate all possible parameters for both exercise safety and effectiveness. Panelists were provided with a framework that identified the patient population (COPD), the setting (acute care, AECOPD, nonintubated or invasively ventilated) and the purpose of the procedure (identify the parameters for safe and effective activity in hospitalized patients with AECOPD). Panelists were given a glossary of working definitions and a list of items developed from the SR to consider. Questions were similarly worded in appropriate language for clinicians, patients and academics. Panelists were first asked what level of consensus must be required among them for an item to be considered an important 'safety' or 'effectiveness' parameter. The panelists were asked to identify what parameters and thresholds were important to identify safe exercise, and what parameters and thresholds were important to identify effective exercise. On entering an item, the panelists were encouraged to identify a threshold for that item, or state "don't know". In this way, the panelist was encouraged to not just identify, for example, "high blood pressure" but was requested to identify a value for what constitutes "high".

The purpose of the second round was to collate the responses with the first round and to invite panelists to review these responses as structured feedback. Participants reviewed their response, the proportion of respondents who answered the same and the majority response. Participants confirmed that their responses for round 1 were adequately summarized, and were given the chance to change or add to their responses.

Round 3 focused on identifying the priority of each item, and the feasibility for its practical use and implementation in a typical Canadian hospital. For each safety and effectiveness parameter, panelists were asked to decide whether an item was 'High Priority', and indicate whether it was a feasible parameter for hospital use. Panelists had the opportunity to make comments or suggest revisions on any of the items listed.

Based on the results from the final round and the information from the SR-SR, a clinical decision-making tool for health care practitioners was developed, as well as an accompanying patient guide.

\section{Next steps}

Critical appraisal through the sensibility assessment: A sensibility assessment of the clinical decision-making tool and accompanying patient guide will be conducted. In addition to a thorough review by the clinicians on the study team, a focus group of physicians, physiotherapists, nurses and respiratory therapists will be convened. They will be asked to review the tool and complete a sensibility questionnaire adapted from the work of Rowe and Oxman (10). The sensibility questionnaire will evaluate the purpose and framework, design, content and face validity, and ease of use on a seven-point Likert Scale. Based on their responses, the clinical decision-making tool will be altered accordingly. Similarly, members of the COPD Canada Patient Network will be invited to review the patient guide and complete a second sensibility questionnaire, with questions on understandability, design, ease of use and other feedback. The tool and guide will be made available in a variety of formats based on the feedback from the health care provider and patient end-users. These formats could include paper copies suitable for lamination, smartphone apps and web-based documents.

\section{The dissemination plan}

The goal for the present project was to provide acute care health care professionals with a simple and clear decision-making tool and an accompanying guide suitable for distribution to COPD patients. The capacity to target multiple national health professional and patient audiences with this network exists. It is well recognized that the sole development and publication of guidelines in peer-reviewed journals does not substantially impact practice. Consequently, a plan structured on concepts derived from the science of KT (implementation science) is required to guide the effective dissemination, implementation and 
evaluation. This KT plan should accompany the development of any practice guideline or other clinical decision-making tool.

Our KT plan for the dissemination, implementation and evaluation of a tool for the safe and effective prescription of exercise for AECOPD patients is in accordance with the framework and Action Cycle described in CIHR's KT handbook "Knowledge Translation in Health Care: Moving from Evidence to Practice" (11).

Figure 1 includes the components of the proposed KT dissemination plan. After identifying the key messages, we will identify the key target groups. Each target group has potential barriers and facilitators for uptake of the tool and guide. For example, for many groups, there may be a lack of knowledge in the area, while for other groups, there may be a lack of confidence in the ability to use the tool and guide effectively. Dissemination tools and strategies will be developed to address the barriers identified, and will include traditional KT strategies such as presentations and publications, as well as more novel approaches such as the support of clinical leaders within institutions. Evaluation methods for each KT strategy is also a key feature of the plan.

\section{DISCUSSION}

Poor activity tolerance due to AECOPD is an independent risk factor for both future hospital admissions and increased mortality (3). There are different interventions that may be used to improve activity tolerance in AECOPD. These include passive mobilization; neuromuscular stimulation, supervised and unsupervised walking within the hospital, and encouraging participation in hospital activities and programs. Exercise therapy is another component of inpatient treatment for AECOPD and has been shown to be efficacious in the research setting; however, it is not systematically implemented in the hospital setting. While there are likely many reasons for this gap, one possibility is the lack of clear and concise guidelines outlining the parameters of safe and effective exercise therapy. In the absence of such guidelines, physicians, physiotherapists and other health care professionals are liable to err on the side of caution and adopt a relatively conservative 'activity as tolerated' approach to prescribing appropriate activity levels rather than prescribing and implementing more structured exercise programs directed at individual physical limitations. This may result in inadequately addressing the existing poor activity tolerance of patients on discharge, potentially worsening patient outcomes and ultimately increasing risk of readmission. This knowledge gap will be assessed by the development an evidence-informed clinical decisionmaking tool and accompanying patient guide based on high-quality sources of information.

A Cochrane systematic review (4) published in 2009 examined the effects of 'early' pulmonary rehabilitation (ie, during or shortly after an AECOPD) on future hospital admissions and other patient-related

\section{REFERENCES}

1. Benady S. The Human and Economic Burden of COPD: A Leading Cause of Hospital Admission in Canada. Ottawa: Canadian Thoracic Society, 2010.

2. Mittmann N, Kuramoto L, Seung SJ, Haddon JM, Bradley-Kennedy C, FitzGerald JM. The cost of moderate and severe COPD exacerbations to the Canadian healthcare system. Respir Med 2008;102:413-21.

3. Garcia-Aymerich J, Farrero E, Felez MA, Izquierdo J, Marrades RM, Anto JM; on behalf of the EFRAM investigators. Risk factors for readmission to hospital for a COPD exacerbation: A prospective study. Thorax 2003;58:100-5.

4. Puhan MA, Gimeno-Santos E, Scharplatz M, Troosters T, Walters EH, Steurer J. Pulmonary rehabilitation following exacerbations of chronic obstructive pulmonary disease. Cochrane Database Syst Rev 2011;(10):CD005305.

5. Kujala UM. Evidence for exercise therapy in the treatment of chronic disease based on at least three randomized controlled trials - summary of published systematic reviews. Scand J Med Sci Sports 2004;14:339-45. outcomes, such as mortality, health-related quality of life and exercise capacity, in patients with COPD. The authors reported that early inpatient pulmonary rehabilitation reduced the risk for hospital admissions, decreased mortality and improved health-related quality of life. There was also a marked improvement in exercise capacity. However, it would be very difficult for health care professionals to develop a safe, practical and effective exercise prescription based on the information that currently exists in the published literature. Specifically, the limitations of the previous work include: lack of inclusion or reporting of safety parameters; limited detail on exercise prescription; and patient population in intervention studies may be too restrictive. Based on these factors, the development of guides for patients and practitioners is warranted.

Our clinical decision-making tool and patient guide have the potential of changing clinical practice and improving patient outcomes. This will only be possible if stakeholders believe the tool is relevant and feasible, the tool is meaningful, and the health care providers and patients actually use it to facilitate change in daily practice. Developing resources to guide clinical practice, in close collaboration with patients, practitioners and researchers, will enhance the credibility of these documents and will increase the likelihood of adoption into clinical practice. In addition, the process of developing the guides will also help identify the barriers to the implementation in practice, which will inform future work. The dissemination, implementation and evaluation plan will not only guide our work, but could inform KT activities for similar acute care hospital populations. A plan that is developed for and by practitioners, researchers and patients may be more innovative, creative and, ultimately, successful compared with a KT strategy that is developed exclusively by the research community. We will share our plan and our process with other groups via the Canadian Lung Association and the Canadian Physiotherapy Association.

In summary, our team of researchers and knowledge users (including patients) conducted a knowledge synthesis, dissemination, implementation and evaluation project that incorporates evidence from both the published literature as well as expert opinion. This multimethod approach will enable us to produce a relevant systematic review, a decision-making tool that will aid practice and a patient guide that will support patients as they recover from an AECOPD.

ACKNOWLEDGEMENTS: The authors acknowledge the Physiotherapy Association of British Columbia, the COPD Canada Patient Network, Chris Wigley, Beth Witherspoon, Tae il Yoon, Debbie Lam, Colin Beattie, Param Backshi and Paolo Macapagal. Pat Camp is a Michael Smith Foundation for Health Research Clinical Scholar.

6. Lemmens V, Oenema A, Knut IK, Brug J. Effectiveness of smoking cessation interventions among adults: A systematic review of reviews. Eur J Cancer Prev 2008;17:535-44.

7. De Niet GJ, Tiemens BG, Kloos MW, Hutschemaekers GJM. Review of systematic reviews about the efficacy of nonpharmacological interventions to improve sleep quality in insomnia. Int J Evid Based Healthcare 2009;7:233-42.

8. Reid WD, Yamabayashi C, Goodridge D, et al. Exercise prescription for hospitalized people with chronic obstructive pulmonary disease and comorbidities: A synthesis of systematic reviews. Int J Chron Obstruct Pulmon Dis 2012;7:297-30.

9. Shea BJ, Grimshaw JM, Wells GA, et al. Development of AMSTAR: A measurement tool to assess the methodological quality of systematic reviews. BMC Med Res Methodol 2007;7:10.

10. Rowe BH, Oxman AD. An assessment of the sensibility of a quality of life instrument. Am J Emerg Med 1993;11:374-80.

11. Straus SE, Tetroe JM, Graham ID. Knowledge Translation in Health Care: Moving from Evidence to Practice. West Sussex: Wiley Blackwell, 2009. 


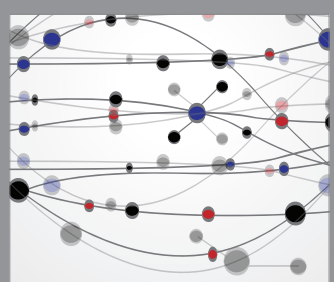

The Scientific World Journal
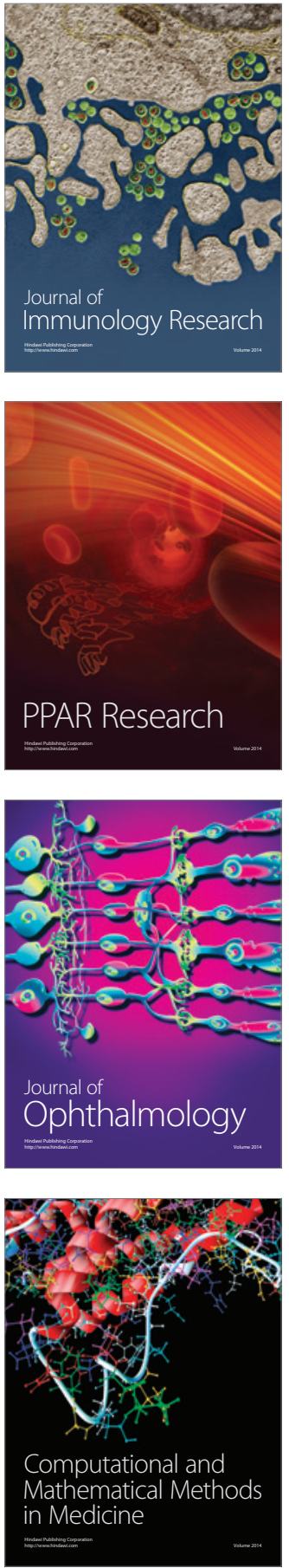

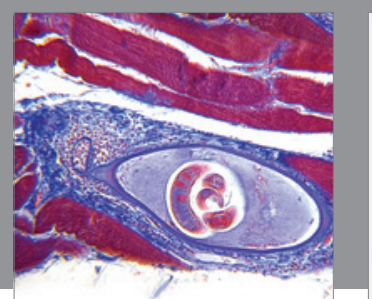

Gastroenterology Research and Practice

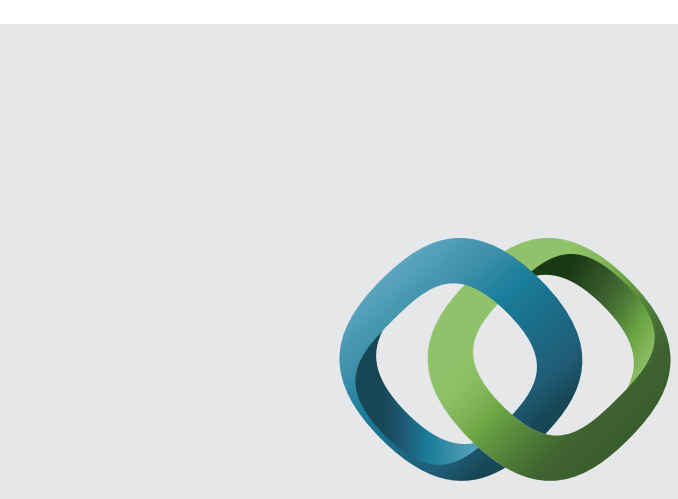

\section{Hindawi}

Submit your manuscripts at

http://www.hindawi.com
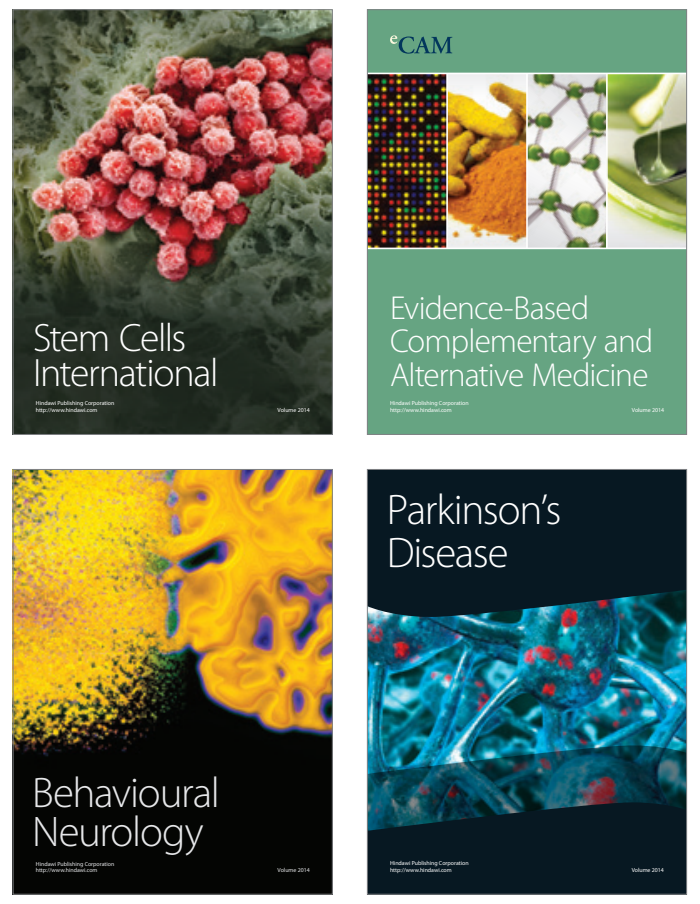
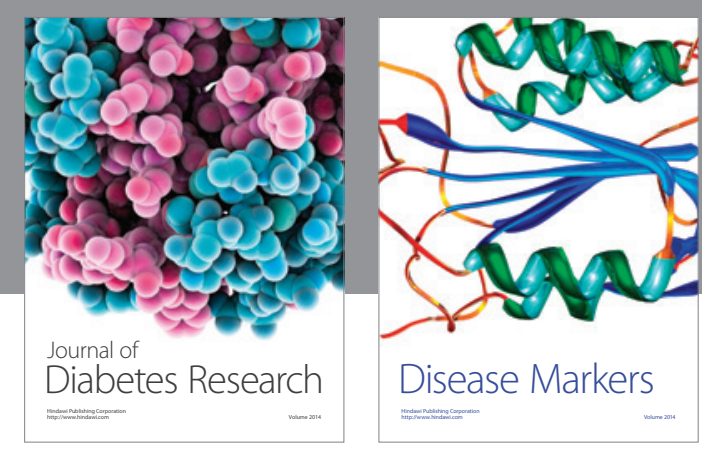

Disease Markers
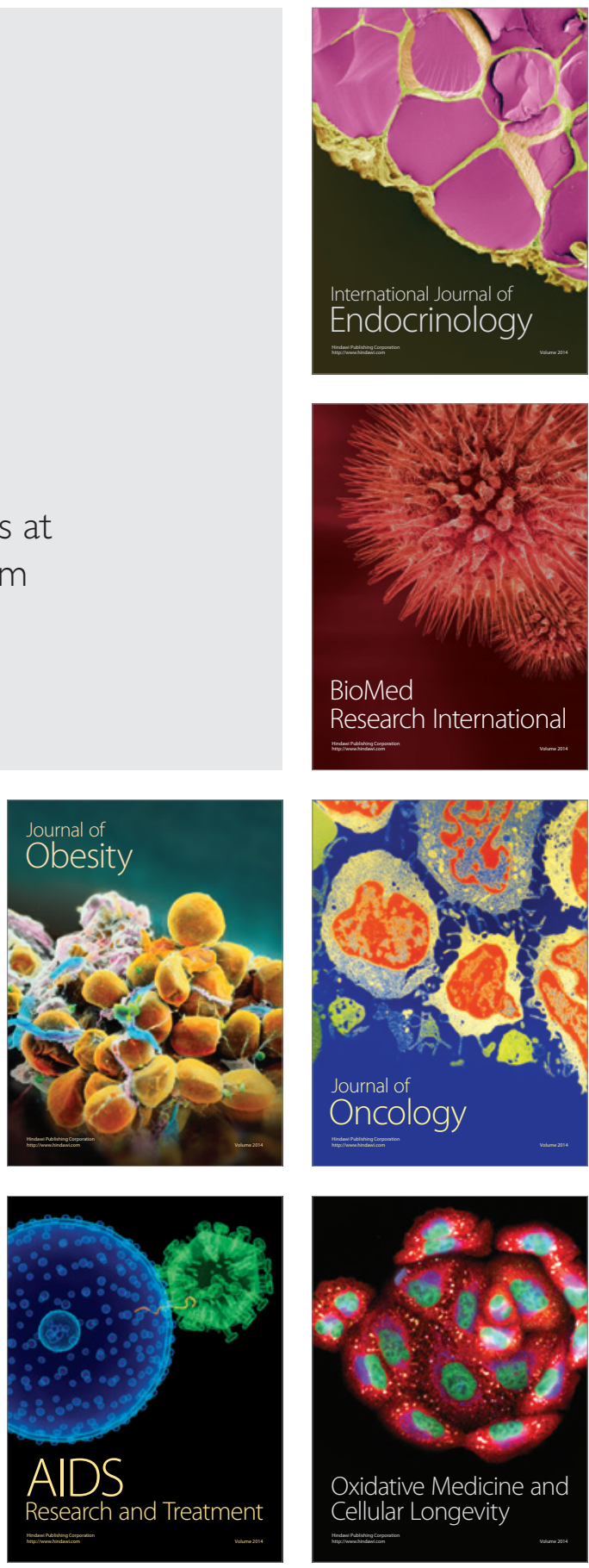